\title{
'Teeth Matter': engaging people experiencing homelessness with oral health promotion efforts
}

\author{
Martha Paisi, ${ }^{* 1}$ Elizabeth Kay, ${ }_{1}^{1}$ Martin Burrows, ${ }^{2}$ Lyndsey Withers, ${ }^{3}$ Anastasios Plessas, ${ }^{1}$ Louisa McDonald ${ }^{2}$ and Robert Witton ${ }^{1,4}$
}

\section{Key points}

Highlights practices that can be used to successfully engage people experiencing homelessness with oral health promotion activities.
Provides a set of resources that can be used in other projects and settings to promote oral health for people experiencing homelessness.
Presents key learning points from the perspective of participants, support workers, clinicians and researchers.

\begin{abstract}
People with experience of homelessness commonly suffer from poor oral health and are likely to have low-level engagement with dental services. 'Teeth Matter' was a pilot study developed to examine whether peer education could result in improved plaque management among people who are experiencing homelessness. Based on the experience gained from developing and implementing the study, and taking into consideration the views of everyone involved, this paper provides a list of suggestions and resources that can be used to improve engagement of this population with oral health promotion activities. Some of the learning points are also applicable in the clinical setting.
\end{abstract}

\section{Background}

Poor dental health is common among people who experience homelessness. ${ }^{1,2}$ A study in London involving 260 people showed that $60 \%$ of the participants had suffered from toothache since they became homeless, while $15 \%$ had pulled out their own teeth. ${ }^{3}$ The British Dental Association ${ }^{4}$ and Groundswell ${ }^{3}$ have highlighted the need to improve oral health care for people experiencing homelessness.

We previously reported on the 'Teeth Matter' research project, the aim of which was to examine whether peer education could yield improved plaque management among people experiencing homelessness. ${ }^{5}$ Along with a

'University of Plymouth, Peninsula Dental School, Plymouth, UK; ${ }^{2}$ Groundswell, Research and Campaigns, London, UK; ' University of Plymouth, Well Connected (charity), Plymouth, UK; ${ }^{4}$ Peninsula Dental Social Enterprise, Plymouth, UK

*Correspondence to: Martha Paisi

Email: martha.paisi@plymouth.ac.uk

Refereed Paper.

Accepted 5 April 2019

DOI: 10.1038/s41415-019-0572-4 demonstration of toothbrushing technique by a dentist, a Groundswell peer educator provided basic oral health messages and used motivational interviewing to develop the participants' sense of self-efficacy regarding oral health. Our participants were 24 British males, aged on average 37 years, who lived in a residential centre providing temporary accommodation to homeless people in Plymouth. ${ }^{5}$

Based on the experience of developing and implementing the project, and considering the views of everyone involved, including our participants, we provide below a list of suggestions and resources that could be helpful to researchers and clinicians when promoting oral health within this community. The paper presents key learning points from people experiencing homelessness, their support workers, researchers and clinicians. Although some of the points raised are also applicable to the general population, we felt that it is important to include them as they can be instrumental in homeless peoples' engagement with oral health promotion efforts. The views were obtained through focus groups discussions and interviews, as well as feedback sheets.

\section{Planning and design}

\section{Participatory approach}

Engaging participants in the design of the study and development of a project ensures that the project will be feasible and acceptable, and that it meets participants' needs. ${ }^{6}$ This is also essential for interesting and engaging participants in the project and making them aware that their views are valued.

\section{Project introduction}

It is important to create interest in the project for participants and in the Teeth Matter study it was effective to host an information session, informally communicating the idea of the planned project to potential participants and responding to any questions they may have. The physical arrangements should provide a sense of equality (for example, sitting in a circle). Having food and drink available to give it a coffee morning feel adds to a relaxed atmosphere. Having a short time interval between the introduction of the project and the baseline assessment (for example, one week rather than a month) is also recommended in order to ensure that participants do not lose 
interest. Advertisement of the project via posters (Appendix 1) and encouragement from support workers are essential for persuading participants to consider joining a project and to maintain their participation.

\section{Outreach work}

Visiting people at a place where they feel comfortable (such as a residential centre for people experiencing homelessness) helps to break down the fears that many of them have regarding dental visits and/or dentists, and facilitates an introduction to the clinical environment in a positive way. When delivering research in a residential centre, you are effectively entering participants' homes, and therefore it is important that the project design takes into account other existing activities in the centre. Furthermore, when advising participants, it is important to consider the constraints they may have in making choices (for instance, limited menu choice in meals provided at the centre) and that there may be a need for establishing partnerships with the top management in order to promote an environment supportive of behavioural change: ${ }^{6}$

'Combining a brief dental check with talking interventions seemed to work for participants. People seemed much more comfortable with taking part in a brief dental check than I expected and I think this may have been because they were on "home ground" (Dentist A);

'The opportunity for first meetings on home territory benefits nervous patients as well as those who accidentally come across the chance to be examined.' (Volunteer, residential homeless centre);

'I believe that the term "hard-to-reach", commonly used to describe homeless people, is not reflective of the actual situation. Based on my overall experience, provided the researchers/ clinicians are open to reaching in to people who experience homelessness and work closely with their support workers, it is easy to establish contact and rapport with them.' (Lead researcher).

\section{Connection with the support service}

Establishing a link with a key person within the support service, whether a day centre or residential, is essential for project development and the recruitment and follow-up of participants. In addition, the involvement of support workers is crucial in establishing rapport between the team and the participants. Improving support workers' awareness regarding oral health as part of the project enables them to better support participants' behaviour change journey:
'Participation rates were higher than I expected and I felt this had a lot to do with the great rapport between the volunteer support worker at the venue and the participants' (Dentist A);

A keylearning has been that health promotion is most effective with homeless people when it works in a joined up way with support services' (Groundswell);

'The assistance of a support worker is paramount to remind them and organise their appointments' (Dentist B);

'Without the lead volunteer's support, I do not think we would be able to reach many (if any) participants, let alone keep them involved in the project. However, adequate support from support workers is needed to help them engage with dental care and highlight the need to keep up with the appointments' (Lead researcher);

'[name of the lead volunteer] told me about you guys, and I need my mouth to be sorted out' (Participant).

\section{Peer researchers}

It is important that peer researchers (that is, people with previous experience of homelessness) are involved throughout the delivery and evaluation of a research/oral health promotion project. Peer researchers ensure that the research tools (for example, information sheets, questionnaires etc) and the language used therein will be acceptable to the participants (for instance, use the word 'project' rather than 'research study'; avoid using the term 'homelessness' but rather refer to 'people in temporary accommodation'). Peer researchers also put residents at ease and connect with them. During qualitative research data collection, such as focus groups, the peer researchers can be accompanied by an academic researcher, who may want to explore certain topics of interest in more depth. Prior to the project, it is also valuable to explore with participants what they would be looking for in a 'peer'; that is, it might go further than being someone who has had personal experience of homelessness, to being someone they can identify with from a wider context, for example, someone from the same city as themselves.

'The presence of a peer researcher was paramount in sparking an interest in the project and discussion, and could be used as a motivation for participants to seek similar recovery from homelessness' (Lead researcher);

'Someone who's been through the same experience. Because if they've been through it, then they can understand it and properly input it to me, maybe in a better way than someone who hasn't lived it' (Participant). ${ }^{5}$

\section{Delivery and implementation}

\section{Relaxed atmosphere}

Music in the background can help make the space a little more relaxed, especially when participants are waiting to be seen by a researcher or the dentist. It can also give an enhanced sense of privacy to participants in discussion with researchers. Provision of snacks and drinks at all study stages (presentation, focus groups, intervention; consumed after the clinical assessment) go down very well and can be used as an opportunity to introduce participants to healthy options:

'I liked the fact that you had refreshments and things like that. All good' (Participant).

\section{First meeting with the dentist}

Some people experiencing homelessness may not have visited a dentist for a significant period of time, and previous negative experiences are common. ${ }^{3}$ More time may be needed for the clinical examination than would be usual in a clinic so that participants have enough time to overcome anxiety, familiarise themselves with the environment and equipment, and to have a chat with the dentist and assistants about their dental journey.

'The participants were diverse and all needed some time to orientate themselves or establish their identity and control over the situation with the dental team, independent of having done this with the other researchers previously. Some started quite serious conversations about their past and future hopes, others needed to play with equipment and some to have a joke about us before they were ready to proceed. Time has to be factored in for participants to make themselves feel secure in their own way, in order to gain their rapport. This may not be so significant at the second visit but some participants may feel they need to update us about their dental appointments for urgent care which will have taken place since the last research visit' (Dentist A).

Reducing waiting time and making the visit 'easy' was appreciated by the participants. A long waiting time can lead to increased anxiety levels:

'In and out, there is no messing around, you don't sit there for hours waiting to be seen. You get in, you fill out the form, by the time you fill up the form you are in there you are seen two minutes later, two minutes later you are done. There is no messing around' (Participant). 
Pain management and urgent referrals

Toothache was common among the study participants. Based on this and the fact that risky behaviours associated with oral mucosa disease, such as smoking, are more prevalent among people experiencing homelessness than in the general population, ${ }^{1}$ it is recommended that, when conducting a research project, a care pathway is arranged in advance to refer participants for urgent/emergency dental problems (for example, pain management, fast track referral form, and GP contact for suspicious lesions).

\section{Opportunity to access treatment}

Providing participants with the opportunity to access treatment as part of a research study is recommended:

'Having a fast track into dental services as with the "Teeth Matter" project proved a great incentive for people to participate, and also meant that people felt they had an opportunity to address their dental need. Once this was in sight, it felt that people felt they could better care for themselves' (Groundswell);

'Participants were primarily interested in getting treatment, especially where they were in pain and/or had difficulty eating' (Volunteer, residential homeless centre);

'Just wanted to get my teeth sorted and get a nice smile back, for my confidence' (Participant).

\section{Conduct}

\section{Communication}

A friendly, informal and non-judgemental atmosphere is important. Giving the time for participants to talk through their concerns (even though sometimes they may not relate to dental care) helps put people at ease:

'It's not frightening going in there. Don't get me wrong the first time I went in there I didn't know what was going to go on...' (Participant);

'It's really important for the clinical team to communicate with the participants in a way which shows respect, friendliness, interest in their lives, some recognition of their current circumstances, without patronising but also appreciating that they may not be used to the dental check-up language and preventive instructions which might be used in a dental practice' (Dentist A);

'I found that they had a need to talk about their previous experiences, good and bad, and the state of their oral health, before going on to more appointments; it helped them process what they were and had been going through, and I found that they responded well to the supported sessions' (Peer educator, Groundswell).

\section{Community engagement}

Engaging with people experiencing homelessness in a non-clinical setting (for instance, volunteering at soup kitchens) helps to understand the issues homeless people face, remove barriers and better engage people. It can also lead to a greater understanding of the issues preventing people from maintaining their oral health. For researchers or clinicians who may have never really spoken to someone who experiences homelessness, having the opportunity to lower that guard away from a research or clinical setting teaches them a new way of approaching and thinking about people experiencing homelessness:

'I think that the long-term involvement of the principal investigator with the local support network for homeless people in Plymouth, including other volunteers such as the volunteer support worker, gave her the ability to develop a protocol which suited the needs and expectations of the participants.' (Dentist A);

'My involvement with a forum providing support to people experiencing homelessness in Plymouth gave me the opportunity to become aware of the challenges that people who are homeless face in their everyday lives and to appreciate the need for flexibility and understanding when it comes to their engagement with the services. Even though I do not have a clinical background, it is very clear that poor oral health is one of the most prevalent issues affecting people experiencing homelessness and this has a significant impact both on their confidence but also their ability to enjoy a meal without pain. Having had prior experience working with people experiencing homelessness enabled me to be able to keep an open mind to specific requirements that this project may have had and to feel comfortable when engaging with our participants' (Lead researcher).

\section{Flexibility to accommodate complex} needs

While some participants valued having set times for study appointments, a high level of flexibility is needed to accommodate anxiety ('please see me as soon as you can') or difficulty with timekeeping and participants' diverse needs. Participants' changing circumstances mean that there should be enough flexibility in the project design to accommodate their attendance behaviours. Furthermore, it is important for researchers to consider changing the study protocol if the change would be beneficial to the participants.

'Structure can help people who experience homelessness to feel more secure and become more able to better organise other aspects of their lives. At the same time, flexibility from the service provision and greater understanding of the need to adjust certain procedures is also important in meeting complex needs' (Lead researcher);

'It may take more than one attempt to get someone to a treatment appointment; encouragement works for some but deters others. No two people are the same' (Volunteer, residential homeless centre);

'Routine, I've got to go down and sort out about work, so I want to go and sort out about my teeth. You know what I mean. It's all routine with me, its stability' (Participant).

\section{Resources}

\section{Customising printed resources}

Discussion with the participants before their involvement in the project is a good way to elicit any questions they have regarding oral health. Subsequent leaflets with information on their questions can be used to provide accurate information on the particular topics and demonstrate to participants that their questions were considered. It was evident through the focus groups that participants had questions regarding several 'myths' widespread through the internet and social media with regards to oral health (for example, the meaning of coloured stripes at the bottom of the toothpaste tubes).

'I have seen online that you've got different tubes for toothpastes and you've got different colours. And they all mean different stuff. Which one to use?' (Participant);

'Which toothpaste is the best to use? Because there is so many different types now' (Participant);

'Like for sensitive teeth, which one is best to use?' (Participant);

'Information on other home treatments, like are teeth whitening kits good for you?' (Participant).

\section{Visual aids}

Visual displays are particularly useful when delivering an oral health intervention to people experiencing homelessness. Messages on an intervention, such as brushing teeth twice a day, can be reinforced with the use of posters or other visual aids at places 
frequented by residents (for instance, in communal bathrooms). Within a common 'look', changing the content of the posters periodically has proven useful in retaining participants' attention. Keeping messages simple and short works well with the particular group (Appendix 2).

'Most of us are learning visually' (Participant);

'They can have a poster at the reception, there is a notice board there. They can do it in ways that stands out too. Not to look like a basic poster' (Participant).

\section{Reminders}

Encouragement and reminders to attend future appointments are important because sometimes chaotic lifestyles can make it difficult for people experiencing homelessness to remember appointments. Whether at the research or clinical setting, provision by support workers of reminder slips which include the study logo, the name of the patient and time of appointment can be used to remind participants of their appointments (Appendix 3). The importance of attending their appointment or informing in advance of their inability to attend can be included in the note. The use of stickers that participants can place somewhere in their room visible to them is recommended, as well as verbal reminders by support workers. Having support workers accompanying patients to their appointment can enhance attendance:

'Reminders would be helpful. If [the volunteer] could give me a tiny 24 hours' reminder on the day before and again before just and if she can knock on my door. I don't always keep appointments because I don't always remember. So if I got the same sticker, I can put in a calendar or just stick it on me' (Participant);

'I'd love a reminder please' (Participant).

\section{Dress code}

The coordinator and researchers can be dressed relatively casually. However, for the dentist and data recorder, scrub uniforms can help give legitimacy as professionals in the eyes of participants.

\section{Goody bags}

Participants highly valued the provision of goody bags. Although the provision of toothpastes and toothbrushes does not guarantee improvement in oral health habits, ensuring that participants have the necessary resources is crucial. Goody bags can contain toothpastes, toothbrushes, timers and laminated information leaflets:

'Reading the information that you gave me a couple of months back helped me with brushing teeth' (Participant);

'You concentrate on the brushing and try to count in your head as well' (Participant).

\section{Acknowledgement for contribution}

Giving out certificates and/or thank-you cards to the participants is a way of acknowledging their contribution to the project and was highly appreciated by our participants (Appendix 4).

\section{Conclusions}

'Teeth Matter' was a pilot project which enabled the team to gain a deeper understanding of the needs of people experiencing homelessness with regard to their oral health. Important lessons that could be used to promote their engagement with oral health promotion have also been obtained. Some of the learnings from this study may also be useful in creating a more inclusive dentistry.

Overall, our experience has shown that oral health does matter to this community. We also believe that the term 'hard to reach', often used to describe people who are homeless, needs to be reconsidered. Small and personal success stories should not be overlooked; a great achievement reported during the project was the fact that one of our participants brushed his teeth for the first time in two years. Such small steps can be signs of emerging confidence and self-efficacy, and such gains can offer pathways to lead to a more stable and healthy lifestyle.

\section{Acknowledgements}

The authors would like to thank the Oral and Dental Research Trust for providing funding for this project (GSK Research Award). We would also like to thank Zoe Allen and Stephan Morrison, for their contribution to the study. A very big thank you to the residential centre's lead volunteer, and to our participants for their participation in the study and who helped expand our knowledge on engaging people who experience homelessness with dental care.

\section{References}

1. Coles $E$, Edwards $M$, Elliot $G M$, Freeman $R$, Heffernan A, Moore A. The oral health of homeless people across Scotland: Report of the homeless oral health survey in Scotland, 2008-2009. Dundee: University of Dundee Dental Health Services Research Unit, 2011. Available at https://dentistry.dundee.ac.uk/sites/dentistry.dundee.ac.uk/ files/smile4life_report2011.pdf (accessed July 2019).

2. Daly B, Newton T, Batchelor P, Jones K. Oral health care needs and oral health-related quality of life (OHIP-14) in homeless people. Community Dent Oral Epidemiol 2010 38: $136-144$

3. Groundswell. Healthy Mouths: A peer-led health audit on the oral health of people experiencing homelessness. London: Groundswell, 2017. Available at https:// groundswell.org.uk/wp-content/uploads/2017/10/ Groundswell-Healthy-Mouths-Report-Final.pdf (accessed July 2019).

4. British Dental Association. Dental care for homeless people. 2003.

5. Paisi M, Witton R, Burrows M et al. Management of plaque in people experiencing homelessness using 'peer education': a pilot study. Br Dent J 2019; 226: 860-866.

6. Akbar T, Turner S, Themessl-Huber M, Freeman R. Evaluation of HMP Shotts Oral Health Improvement Project. Dundee: University of Dundee Dental Health Services Research Unit, 2012. Available at https:// dentistry.dundee.ac.uk/sites/dentistry.dundee.ac.uk/ files/ESOP\%20Report\%20July\%202011.pdf (accessed July 2019). 


\section{Teeth Matter}

\section{Participants needed Gan you help?}

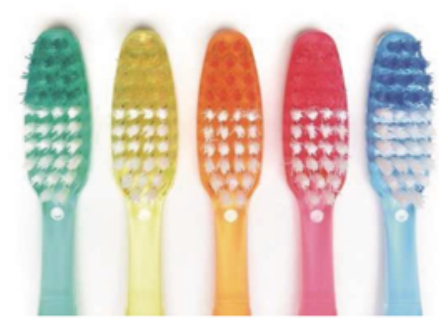

Peninsula Dental School and the charity Groundswell will be running an informal session to tell you about a project on looking after your teeth

\section{Come along and join us for discussion and refreshments}

Organisations' logos

Appendix 1 'Teeth Matter' poster

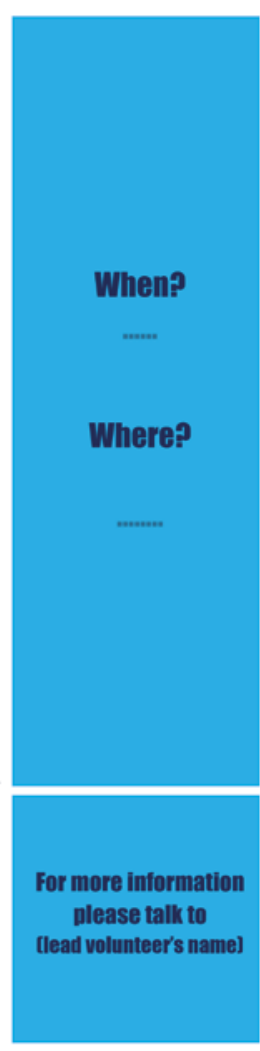

\section{Dental Top Tips for a Healthy You}

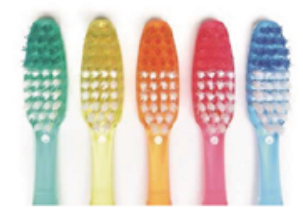

$*$ Brush your teeth twice a day. Always last thing at night and one other time during the day.

* Use toothpaste with fluoride $(1,350-1,500 \mathrm{ppm}$ fluoride).

$\leftarrow$ It is recommended that you brush for $\mathbf{2}$ minutes.

* Try not to rinse your mouth with water after you brush your teeth. This will wash the fluoride off your teeth.

* Cut down on sugary food and drinks and try to have them only! with meals.

* If you visit a dentist regularly, you can check that both your teeth and mouth are healthy.

A healthy mouth is an important part of your overall health

Organisation's logo

Organisation's logo

Appendix 2 'Dental Top Tips for a Healthy You' poster

\begin{tabular}{|c|c|c|c|c|}
\hline Teeth Matter & Dental appointment & \multicolumn{3}{|c|}{ Date (e.g. Friday 25 ${ }^{\text {th }}$ May) } \\
\hline GENTLE & $\begin{array}{c}\text { GEMINDER } \\
\text { name }\end{array}$ & Location & Time & $\begin{array}{c}\text { Please attend to } \\
\text { keep your } \\
\text { eligibility for } \\
\text { treatment }\end{array}$ \\
\hline
\end{tabular}

Appendix 3 Reminder of appointment note

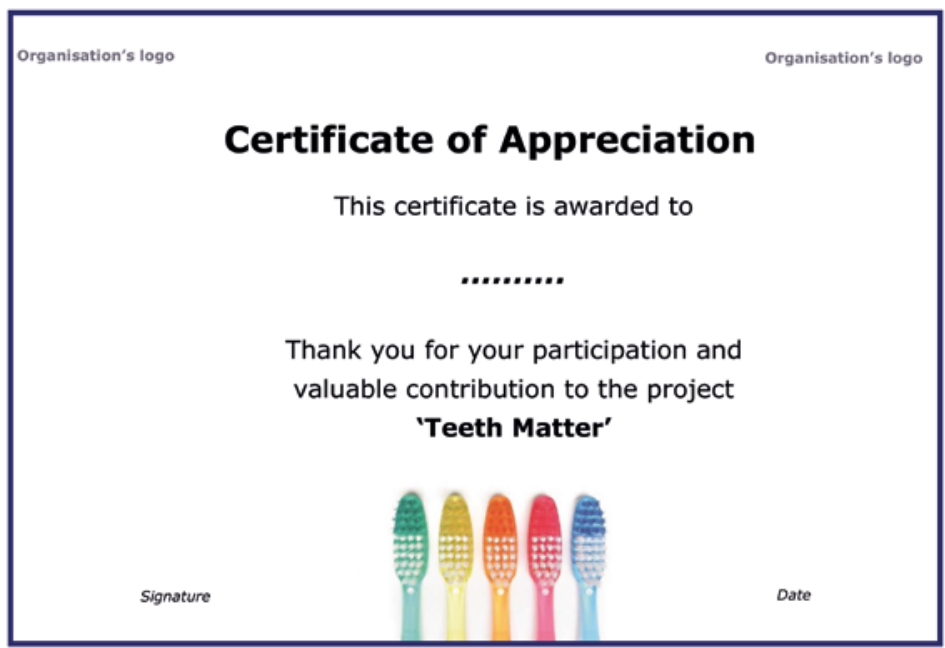

Appendix 4 Certificate of Appreciation 